\title{
Oligomeric States and Hydrodynamic Properties of Lysyl Oxidase-Like 2
}

\author{
Alex A. Meier ${ }^{1}$, Hee-Jung Moon ${ }^{1, \dagger}{ }^{,}$Ronald Toth IV ${ }^{2, \ddagger}$, Ewa Folta-Stogniew ${ }^{3}$, Krzysztof Kuczera ${ }^{1,4} \mathbb{D}_{\text {, }}$ \\ C. Russell Middaugh ${ }^{2}$ and Minae Mure ${ }^{1, *}$
}

1 Department of Chemistry, University of Kansas, Lawrence, KS 66045, USA; aameier@ku.edu (A.A.M.); hjmoon@ku.edu (H.J.M.); kkuczera@ku.edu (K.K.)

2 Department of Pharmaceutical Chemistry, School of Pharmacy, University of Kansas, Lawrence, KS 66047, USA; ronald.toth@gmail.com (R.T.IV); middaugh@ku.edu (C.R.M.)

3 W.M. Keck Biotechnology Resource Laboratory, Department of Molecular Biophysics and Biochemistry, Yale School of Medicine, New Haven, CT 06511, USA; ewa.folta-stogniew@yale.edu

4 Department of Molecular Biosciences, University of Kansas, Lawrence, KS 66045, USA

* Correspondence: mmure@ku.edu

+ Current address: Department of Pharmacology and Toxicology, University of Kansas, Lawrence, KS 66045, USA.

$\ddagger$ Current address: Characterization, Genomic Medicine Unit, Sanofi, Waltham, MA 02451, USA.

check for

updates

Citation: Meier, A.A.; Moon, H.-J.; Toth, R., IV; Folta-Stogniew, E.; Kuczera, K.; Middaugh, C.R.; Mure, M. Oligomeric States and Hydrodynamic Properties of Lysyl Oxidase-Like 2. Biomolecules 2021, 11, 1846. https://doi.org/10.3390/ biom 11121846

Academic Editors: Katalin Csiszar and Eugene A. Permyakov

Received: 3 November 2021 Accepted: 4 December 2021 Published: 8 December 2021

Publisher's Note: MDPI stays neutral with regard to jurisdictional claims in published maps and institutional affiliations.

Copyright: (c) 2021 by the authors. Licensee MDPI, Basel, Switzerland. This article is an open access article distributed under the terms and conditions of the Creative Commons Attribution (CC BY) license (https:/ / creativecommons.org/licenses/by/ $4.0 /)$.

\begin{abstract}
Lysyl oxidase-like 2 (LOXL2) has emerged as a promising therapeutic target against metastatic/invasive tumors and organ and tissue fibrosis. LOXL2 catalyzes the oxidative deamination of lysine and hydroxylysine residues in extracellular matrix (ECM) proteins to promote crosslinking of these proteins, and thereby plays a major role in ECM remodeling. LOXL2 secretes as 100-kDa fulllength protein (fl-LOXL2) and then undergoes proteolytic cleavage of the first two scavenger receptor cysteine-rich (SRCR) domains to yield 60-kDa protein ( $\triangle 1-2$ SRCR-LOXL2). This processing does not affect the amine oxidase activity of LOXL2 in vitro. However, the physiological importance of this cleavage still remains elusive. In this study, we focused on characterization of biophysical properties of fl- and $\triangle 1$-2SRCR-LOXL2s (e.g., oligomeric states, molecular weights, and hydrodynamic radii in solution) to gain insight into the structural role of the first two SRCR domains. Our study reveals that fl-LOXL2 exists predominantly as monomer but also dimer to the lesser extent when its concentration is $<\sim 1 \mathrm{mM}$. The hydrodynamic radius $\left(R_{\mathrm{h}}\right)$ determined by multi-angle light scattering coupled with size exclusion chromatography (SEC-MALS) indicates that fl-LOXL2 is a moderately asymmetric protein. In contrast, $\triangle 1-2 S R C R-L O X L 2$ exists solely as monomer and its $R_{\mathrm{h}}$ is in good agreement with the predicted value. The $R_{\mathrm{h}}$ values calculated from a 3D modeled structure of fl-LOXL2 and the crystal structure of the precursor $\triangle 1-2 S R C R-L O X L 2$ are within a reasonable margin of error of the values determined by SEC-MALS for fl- and $\triangle 1-2$ SRCR-LOXL2s in mature forms in this study. Based on superimposition of the 3D model and the crystal structure of $\triangle 1-2 \mathrm{SRCR}-\mathrm{LOXL2}$ (PDB:5ZE3), we propose a configuration of fl-LOXL2 that explains the difference observed in $R_{\mathrm{h}}$ between fl- and $\triangle 1-2 S R C R-L O X L 2 s$ in solution.
\end{abstract}

Keywords: lysyl oxidase-like 2; scavenger receptor cysteine-rich; extracellular matrix; hydrodynamic radius; analytical ultracentrifugation; isoelectric points

\section{Introduction}

Lysyl oxidase-like 2 (LOXL2) is a member of the LOX-family of proteins that are lysine tyrosylquinone (LTQ)- and copper (II)- dependent amine oxidases. The LTQ cofactor is post-translationally derived from the conserved Tyr and Lys residues in the active site [1,2]. LOXL2 oxidatively deaminates lysine and hydroxylysine residues in extracellular matrix (ECM) proteins such as tropoelastin and collagen type IV to initiate their crosslinking and promote ECM remodeling [3-5]. In addition to the traditional ECM substrates, platelet growth factor receptor $\beta$ (PDGFR $\beta$ ) was recently identified as a cell-surface substrate of 
LOXL2 [6]. Elevated expression of LOXL2 has been associated with poor prognosis in metastatic/invasive cancers and fibrotic disorders [7-9]. Small molecule inhibitors and inhibitory RNAs have shown to effectively retard disease progression in both in vitro and in vivo studies [6,9-16]. Despite its prominence as a therapeutic target, little progress has been made on structure-based drug design for LOXL2 specific inhibitors because of the lack of 3D structural information of the catalytically competent (mature) form of LOXL2.

The loxl2 gene encodes four scavenger receptor cysteine-rich (SRCR) domains at the $\mathrm{N}$-terminus and the amine oxidase domain at the C-terminus (Figure 1). The C-terminal amine oxidase domain is conserved among the LOX-family of proteins and the catalytic domains of LOXL2 and LOX share $68 \%$ similarity and $49 \%$ identity. SRCR domains are ancient motifs found on the cell surface and are involved in protein-protein, proteinsubstrate or receptor-ligand interactions $[17,18]$. SRCR domains are $90-110$ amino acids long and contain highly conserved Cys residues. Based on the numbers of Cys residues and intradomain disulfide bonds, SRCR domains are classified into two categories (type A and type B). Type A domain is characterized by six Cys residues (three disulfide bonds) while type B domain is characterized by eight Cys residues (four disulfide bonds). The SRCR domains of LOXL2 contain six Cys residues per domain, therefore it is categorized as type A [17]. The physiological role of SRCR domains of LOXL2 largely remains elusive.

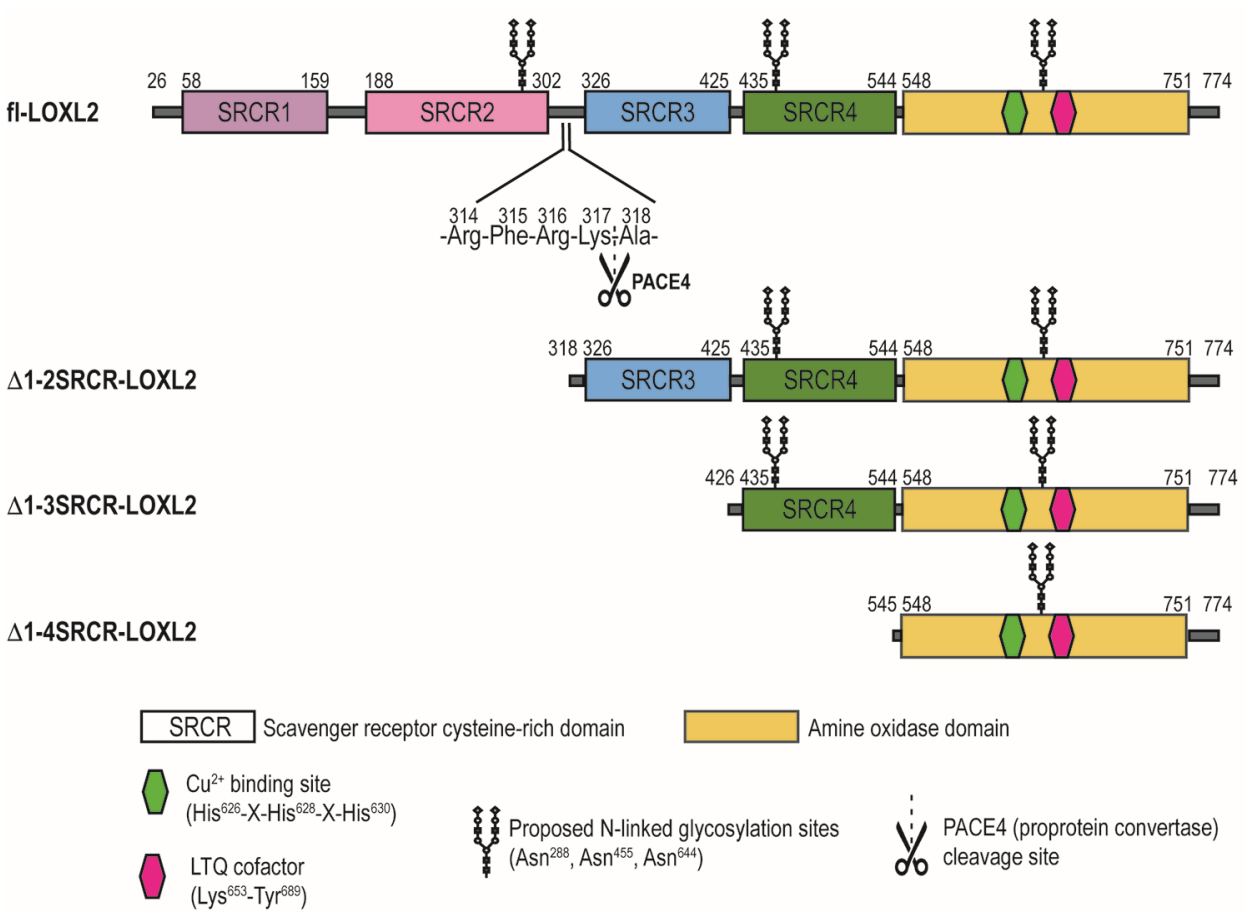

Figure 1. A schematic diagram of rLOXL2s in this study. fl-LOXL2: full-length LOXL2; $11-2 \mathrm{SRCR}-$ LOXL2: LOXL2 which lacks the first two SRCR domains; 1 1-3SRCR-LOXL2: LOXL2 which lacks the first three SRCR domains; 1 1-4SRCR-LOXL2: LOXL2 which lacks all four SRCR domains.

LOXL2 secretes as 100-kDa full-length protein (fl-LOXL2) and that undergoes proteolytic cleavage of the first two SRCR domains by PACE4, a proprotein convertase, at $\mathrm{Arg}^{314}-\mathrm{Phe}^{315}$ - $\mathrm{Arg}^{316}-\mathrm{Lys}^{317} \downarrow \mathrm{Ala}^{318}$ (underlined: the recognition sequence of PACE4) to yield $\sim 60-\mathrm{kDa}$ protein ( $\triangle 1-2 \mathrm{SRCR}-\mathrm{LOXL2)}$ [4]. In vitro, this processing does not affect the amine oxidase activity of LOXL2 in the oxidation of cadaverine and tropoelastin, but increases the solubility of LOXL2 [4]. The physiological significance of this processing is still unclear. In 2018, a $2.4 \AA$ crystal structure of a $\mathrm{Zn}^{2+}$-bound precursor form (lacking the LTQ cofactor) of $\triangle 1-2$ SRCR-LOXL2 became available [19]. The overall structure of $\triangle 1$-2SRCR-LOXL2 is in a triangular shape (Figure 2A) where the 3rd SRCR domain interacts with the catalytic domain through in total of six hydrogen bonds and two van der Waals interactions (Figure 2E). The 4th SRCR domain has no interaction with either the 
catalytic domain or the $3^{\text {rd }}$ SRCR domain (Figure 2C,D). The precursor residues of LTQ (Lys653 and Tyr689) were $16.6 \AA$ apart (Figure 2B) and it was suggested that a substantial conformational change is required to enable the LTQ cofactor formation.

(A)

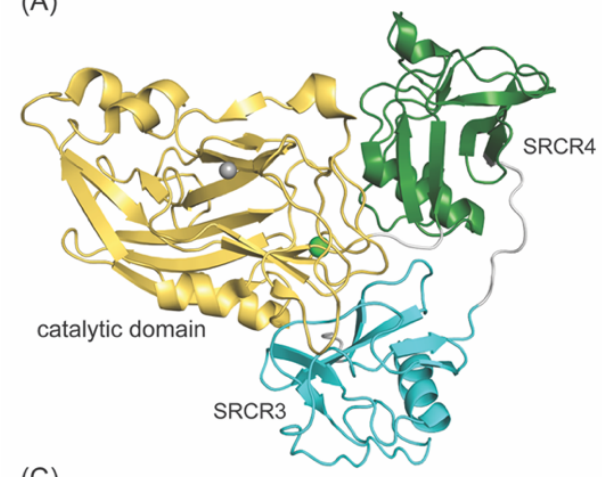

(C)

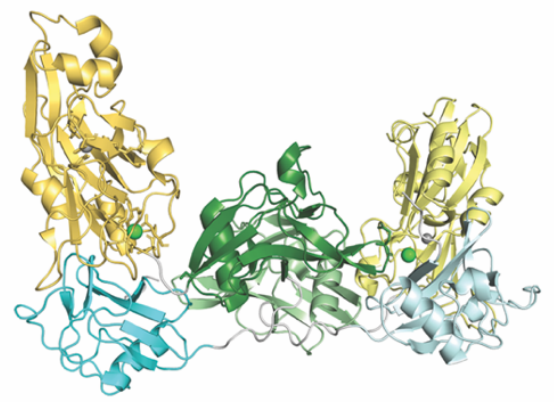

(E)

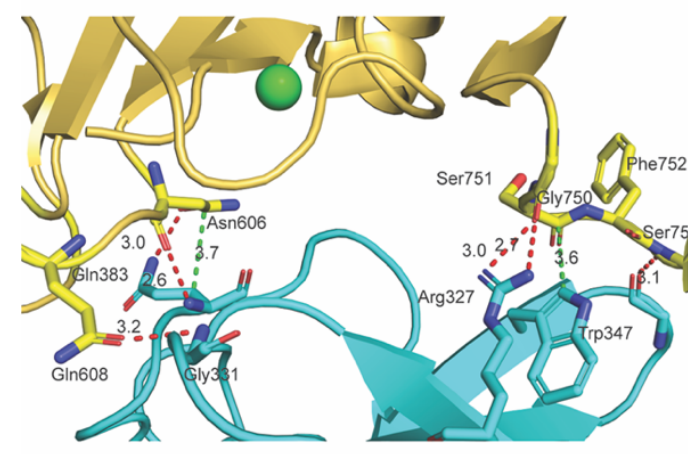

(B)

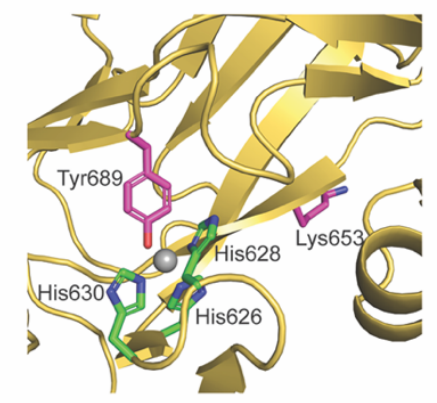

(D)

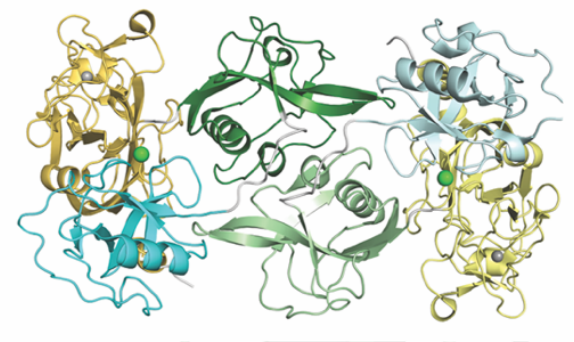

(F)

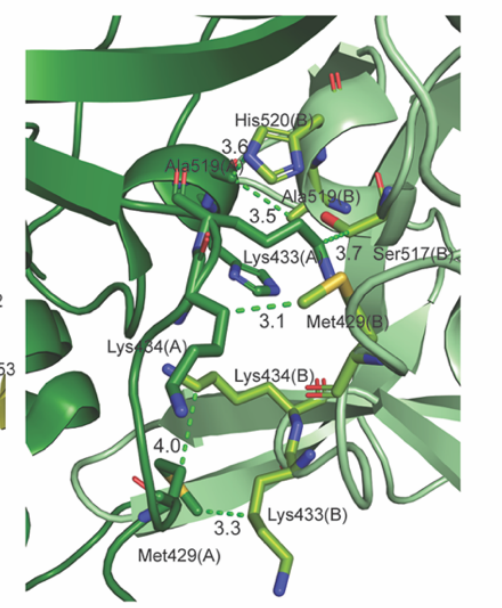

Figure 2. X-ray structure of $\triangle 1$-2SRCR-LOXL2 in the precursor form (PDB: 5ZE3). (A) Monomer (B) The active site structure. The precursor residues (Lys653, Tyr689) are $16.6 \AA$ apart. $\mathrm{Zn}^{2+}$ (gray sphere) occupies the predicted $\mathrm{Cu}^{2+}$ binding site (His626-X-His628-X-His630). $\mathrm{Ca}^{2+}$ is shown as a green sphere. (C) In each asymmetric unit (ASU), two molecules are observed. The intermolecular association between monomers is mediated mainly through the 4th SRCR domains (in forest and light green). (D) A top view of the ASU shown in (C). (E) The 3rd SRCR domain (in cyan) interacts with the catalytic domain (in yellow) mostly through hydrogen bonding interactions (in red) but also through van der Waals interactions (in green). (F) The intermolecular association observed in between the 4th SRCR domains (shown in C,D) are all through van der Waals interactions (in green). Numbers in $(\mathbf{A}, \mathbf{E}, \mathbf{F})$ are all in $\AA$.

In this study, we focused on understanding the biophysical significance of the proteolytic cleavage of the first two SRCR domains using catalytically competent forms (containing the LTQ cofactor and $\mathrm{Cu}^{2+}$ ) of full-length (fl-LOXL2) and 60-kDa form $(\triangle 1-2 S R C R-$ LOXL2) of LOXL2. The latter was generated from K317R mutant (furin-cleavage site, ${ }^{315}$ Arg-Phe- ${ }^{316}$ Arg- ${ }^{317}$ Arg, was engineered) of LOXL2. We determined the oligomeric states and hydrodynamic radii $\left(R_{\mathrm{h}}\right)$ of the two forms of LOXL2. The latter values are 
compared with those calculated from a 3D model structure of the precursor fl-LOXL2 being generated by AlphaFold V2 [20] and the crystal structure of the precursor $\triangle 1-2 \mathrm{SRCR}$ LOXL2 [19], respectively.

\section{Materials and Methods}

\subsection{Protein Purification}

The fl- and $\Delta 1$-2SRCR-LOXL2s were both produced in FreeStyle ${ }^{\mathrm{TM}} 293$ Expression System (Thermo Fisher Scientific, Lenexa, KS, USA) and were purified as described previously [4]. $\triangle 1-3 S R C R$ - and $\triangle 1-4$ SRCR-LOXL2s were produced in DES ${ }^{\circledR}$ system (Thermo Fisher Scientific) and were purified as described previously [21].

\subsection{PAGE Analysis}

For SDS-PAGE, $4 \mu \mathrm{g}$ of protein samples was loaded on to a $10 \%$ TGX $^{\mathrm{TM}}$ FastCast $^{\mathrm{TM}}$ (BioRad Laboratories, Hercules, CA, USA) polyacrylamide gel. Protein samples were prepared with 4 X sample loading buffer (Bio-Rad Laboratories) for reducing conditions and with Pierce $^{\mathrm{TM}}$ LDS sample buffer, non-reducing (4X) (Thermo Fisher Scientific) for non-reducing conditions. For native PAGE, protein samples were loaded on to a NativePAGE ${ }^{\mathrm{TM}} 4$ to 16\% Bis-Tris Mini Protein Gels (Thermo Fisher Scientific) with NativeMark ${ }^{\mathrm{TM}}$ Unstained Protein Standard (Thermo Fisher Scientific) and separated at $125 \mathrm{~V}$ for $2 \mathrm{~h}$. A recombinant prolyl-4-hydroxylase (homodimer, $49.2-\mathrm{kDa}$ ) was used as the protein standard [22].

\subsection{SEC-MALS}

The light scattering data were collected from size exclusion chromatography (SEC) using a Superdex 200 10/300, HR Size Exclusion Chromatography column (GE Healthcare, Piscataway, NJ, USA) connected to Agilent 1200 High Performance Liquid Chromatography (HPLC) System (Agilent Technologies, Wilmington, DE, USA) equipped with an autosampler. The elution from SEC was monitored by a photodiode array (PDA) UV/VIS detector (Agilent Technologies, Wilmington, DE, USA), differential refractometer (OPTILabrEx Wyatt Corp., Santa Barbara, CA, USA), static and dynamic, multiangle laser light scattering (LS) detector (HELEOS II with QELS capability, Wyatt Corp., Santa Barbara, CA, USA). The SEC-UV/LS/RI system was equilibrated in $50 \mathrm{mM}$ Tris, $\mathrm{pH} 8.0$ containing $150 \mathrm{mM} \mathrm{NaCl}$ and $0.01 \%$ sodium azide at a flowrate of $0.5 \mathrm{~mL} / \mathrm{min}$ or $1.0 \mathrm{~mL} / \mathrm{min}$. Two software packages were used for data collection and analysis: the Chemstation software (Agilent Technologies, Wilmington, DE, USA) controlled the HPLC operation and data collection from the multi-wavelength UV/VIS detector, while the ASTRA software (Wyatt Corp., Santa Barbara, CA, USA) collected data from the refractive index detector, the light scattering detectors, and recorded the UV trace at $280 \mathrm{~nm}$ sent from the PDA detector. The weight average molecular masses, Mw, were determined across the entire elution profile in the intervals of $1 \mathrm{~s}$ from static LS measurement using ASTRA software as previously described [23]. Hydrodynamic radii, $R_{\mathrm{h}}$, were measured from an "on-line" dynamic LS measurement every $2 \mathrm{~s}$. The dynamic light scattering signal was analyzed by the method of cumulants [24].

\subsection{Analytical Ultracentrifugation}

Analytical ultracentrifugation (AUC) experiments were performed on an Optima XL-I (Beckman Coulter, Fullerton, CA, USA) analytical ultracentrifuge equipped with a scanning UV-Visible optical system set at $280 \mathrm{~nm}$ and an An-60 Ti 4-hole rotor. Sedimentation velocity experiments were conducted using a Beckman double-sector Epon centerpiece cell with $12 \mathrm{~mm}$ sapphire windows at 40,000 RPM. The experiments were performed in $50 \mathrm{mM}$ HEPES (pH 8.0) containing between $0.088 \mathrm{mg} / \mathrm{mL}$ and $0.88 \mathrm{mg} / \mathrm{mL}$ fl-LOXL2. The data were analyzed using the continuous sedimentation coefficient distribution model $c(s)$ of SEDFIT [25]. Sedimentation equilibrium experiments were performed using a six-sector Epon centerpiece cell with $12 \mathrm{~mm}$ sapphire windows (Beckman Coulter). Rotor speeds of 
$10,000,12,500$, and 15,000 RPM were applied. The data were analyzed using HeteroAnalysis to fit molecular weight, $n, \ln (K)$ and baseline [26].

\section{5. pI Determination of LOXL2s}

Purified recombinant LOXL2s were subjected to 2-dimensional (2D) gel electrophoresis. ReadyStrip ${ }^{\mathrm{TM}}$ IPG Strips (11 cm pH 3-10 nonlinear, immobilized $\mathrm{pH}$ gradient, IPG strip) (Bio-Rad Laboratories) were rehydrated with $200 \mu \mathrm{L}$ of rehydration buffer [ $8 \mathrm{M}$ urea, 2\% CHAPS, 50 mM dithiothreitol (DTT), 0.2\% Bio-Lyte ${ }^{\circledR} 3 / 10$ ampholyte, and $0.001 \%$ Bromophenol Blue] containing $100 \mu \mathrm{g}$ of protein for $12 \mathrm{~h}$ in a rehydration tray overlayed with $5 \mathrm{~mL}$ of mineral oil. The excess mineral oil was removed from the rehydrated strips by blotting the strip on a filter paper. The IPG strips were transferred to the running tray of a PROTEAN $^{\circledR}$ i12 ${ }^{\text {TM }}$ IEF system (Bio-Rad Laboratories) before being overlaid with another 5 $\mathrm{mL}$ of mineral oil and run using the recommended procedure for $11 \mathrm{~cm} \mathrm{pH} \mathrm{3-10} \mathrm{IPG} \mathrm{strips}$ (e.g., 250 volts for $20 \mathrm{~min}, 8000$ volts for $1 \mathrm{~h}$, and 8000 volts for 26,000 volt hours, with a total run time of approximately $6 \mathrm{~h}$ at $20^{\circ} \mathrm{C}$ ). The IPG strips were taken out of the IEF system, and the excess mineral oil was removed by blotting. The strips were then equilibrated with $4 \mathrm{~mL}$ of SDS-PAGE Equilibration Buffer 1 (6 M urea, 2\% SDS, $0.375 \mathrm{M}$ Tris-HCl (pH 8.8), $20 \%$ glycerol and 2\% $(w / v)$ DTT) for 15 min and SDS-PAGE Equilibration Buffer 2 [6 M urea, $2 \%$ SDS, $0.375 \mathrm{M}$ Tris- $\mathrm{HCl}$ (pH 8.8), 20\% glycerol, and $0.135 \mathrm{M}$ iodoacetamide] from the ReadyPrep ${ }^{\mathrm{TM}}$ 2D Starter Kit (Bio-Rad Laboratories) for $15 \mathrm{~min}$. Equilibrated IPG strips were rinsed in 1X TGS running buffer before mounting onto an AnykD ${ }^{\mathrm{TM}}$ Criterion $^{\mathrm{TM}} \mathrm{TGX}$ Stain-Free ${ }^{\mathrm{TM}}$ Precast Gel (Bio-Rad Laboratories) and overlaying with $0.5 \%$ agarose. The $2 \mathrm{D}$ gel was run at $200 \mathrm{~V}$ for $60 \mathrm{~min}$ or until the dye front reached the bottom of the gel. The gels were then stained using Coomassie Brilliant Blue G-250 stain before imaging on a Chemidoc ${ }^{\mathrm{TM}}$ MP Imaging system (Bio-Rad Laboratories). $\mathrm{pI}$ values were calculated based on the length that the protein traveled through the IPG strip.

\section{Results and Discussion}

\subsection{Polyacrylamide Gel Electrophoresis}

Intermolecular association of two molecules of $\mathrm{Zn}^{2+}$-bound precursor $\triangle 1$-2SRCRLOXL2 [19] is mediated mainly through SRCR4 domains via van der Waals interactions (3.3-4.0 A) [27], as shown in Figure 2F. Although it was stated that the precursor $\Delta 1-2 S R C R-$ LOXL2 was monomeric in solution, no experimental data supporting this statement was provided [19]. In this study, both fl-LOXL2 and $\triangle 1-2 S R C R-L O X L 2$ in mature forms were purified to homogeneity (Figure 3A,B). fl-LOXL2 and $\triangle 1-2 S R C R-L O X L 2$ were subjected to polyacrylamide gel electrophoresis (PAGE) under both reducing and non-reducing conditions (Figure 3A). In the presence of a reducing reagent (all disulfide bonds are reduced), the molecular weights of fl-LOXL2 and $\triangle 1-2 S R C R-L O X L 2$ were $\sim 95-\mathrm{kDa}$ and $\sim 60-\mathrm{kDa}$, respectively, which are in good agreement with the predicted values for monomers. In the absence of a reducing agent, both proteins are more compact and migrated as $\sim 20-\mathrm{kDa}$ smaller in size than when a reducing agent was added (Figure 3A) [28].

When these protein samples were subjected to native PAGE, $\triangle 1-2 S R C R-L O X L 2 \mathrm{mi}-$ grated (Figure 3B) similarly to the molecular weight under denaturing and reducing conditions (Figure $3 \mathrm{~A}$ ), suggesting that $\triangle 1-2 \mathrm{SRCR}$-LOXL2 is a monomer. $\triangle 1-3 S R C R$ - and $\triangle 1-4 S R C R-L O X L 2 s$ (Figure 3B) that lack the first three and all of the four SRCR domains also migrated to the expected molecular weights $(42.7-\mathrm{kDa}, 29.6-\mathrm{kDa})$ that were previously determined by mass spectrometry [21]. On the other hand, fl-LOXL2s of the wild-type (WT-LOXL2), R314A/R316A/K317Q-LOXL2 (AAQ-LOXL2, a PACE4 cleavage site null mutant) [4] and K317R-LOXL2 were detected as a single band with molecular weight of $\sim 175-\mathrm{kDa}$ but no protein band corresponding to the monomer, expected to be $\sim 75-\mathrm{kDa}$ under non-reducing conditions (Figure 3A), was detected. In addition to the $\sim 175-\mathrm{kDa}$ band, a faint band with a much higher molecular weight $(\sim 340-\mathrm{kDa})$ was detected in all three lanes containing fl-LOXL2 (AAQ-, WT- and K317R-LOXL2s) (Figure 3B). These results suggest that fl-LOXL2 exists predominantly as dimer and also as tetramer to a lesser 
extent. Since we did not detect dimers or other oligomers for $\triangle 1-2 S R C R-, \triangle 1-3 S R C R-$, and $\triangle 1-4$ SRCR-LOXL2s, it is evident that one or both of the first two SRCR domains is/are essential for oligomerization of fl-LOXL2.

(A)

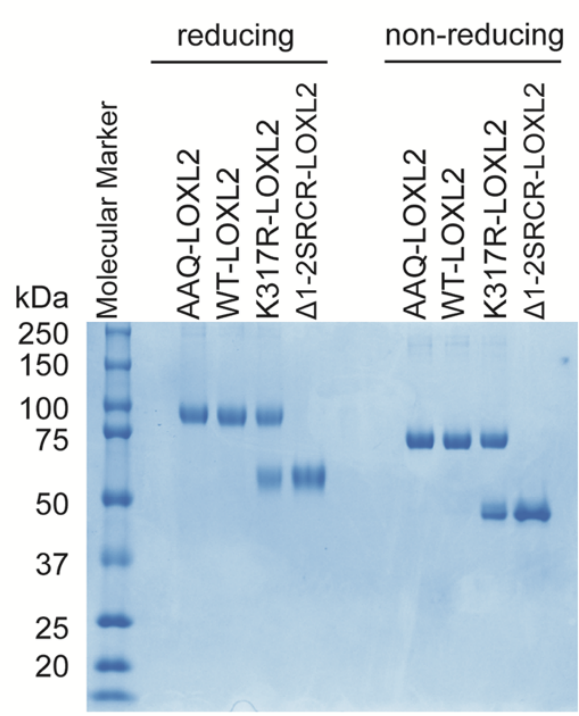

(B)

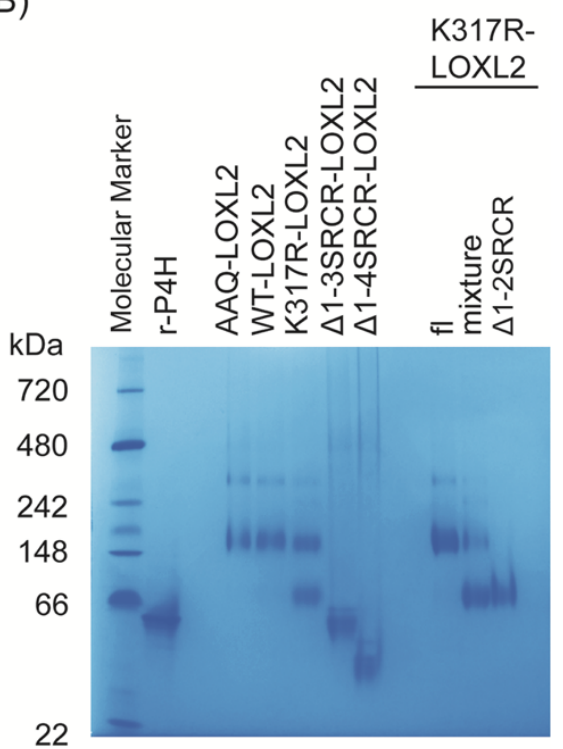

Figure 3. Polyacrylamide gel electrophoresis (PAGE) of LOXL2s. (A) SDS-PAGE under denaturing conditions in the presence and absence of reductant ( $\beta$-mercaptoethanol). (B) Native-PAGE. AAQ: R315A/R316A/K317Q mutant form of LOXL2; WT: wild type; K317R-LOXL2 was isolated as a mixture of fl-LOXL2 and $\triangle 1-2 S R C R-L O X L 2 ; \triangle 1-2 S R C R-L O X L 2$ was isolated from the mixture by FPLC; $\mathrm{r}-\mathrm{P} 4 \mathrm{H}$ : recombinant Bacillus anthracis prolyl-4-hydroxylase as a molecular standard (homodimer, $49.2 \mathrm{kDa})$ [22].

\subsection{Molecular Weight Determination by SEC-MALS}

The molecular masses of fl-LOXL2 and $\triangle 1$-2SRCR-LOXL2 were determined by size exclusion chromatography coupled with multi angle light scattering (SEC-MALS) (Figure 4). At concentrations from 0.08 to $1.6 \mu \mathrm{M}$, fl-LOXL2 exists predominantly as the monomer with molecular weight of $\sim 83-\mathrm{kDa}$ at the elution volume of $13.6 \mathrm{~mL}$ but a small amount of dimer with molecular weight of $\sim 176-\mathrm{kDa}$ at the elution volume of $12.1 \mathrm{~mL}$ and a minor amount of tetramer (unable to calculate the molecular weight) at the elution value of $7.6 \mathrm{~mL}$ (Figure 4A). On the other hand, $\triangle 1-2 \mathrm{SRCR}-\mathrm{LOXL} 2$ elutes solely as a monomer with molecular weight of $\sim 56-\mathrm{kDa}$ at the elution volume of $15.5 \mathrm{~mL}$ (Figure $4 \mathrm{~B}$ ). The amounts of oligomers (dimer, tetramer) in Figure $4 \mathrm{~A}$ in comparison to those in native acrylamide gel electrophoresis (Figure 3B) strongly indicates that fl-LOXL2 is predominantly a monomer but it is in an equilibrium of monomer-dimer-tetramer.

\subsection{The Oligomeric State of fl-LOXL2}

Since some oligomers (e.g., mostly dimers and tetramers in a lesser amount) were observed for fl-LOXL2 in the native PAGE and SEC-MALS (Figures 3B, 4 and 5), oligomerization of fl-LOXL2 was observed by sedimentation velocity-analytical ultracentrifugation (SV-AUC) at $20^{\circ} \mathrm{C}$ with rotor speed at $40,000 \mathrm{RPM}$. Three different concentrations $(0.88 \mathrm{mg} / \mathrm{mL}, 0.30 \mathrm{mg} / \mathrm{mL}$ and $0.088 \mathrm{mg} / \mathrm{mL})$ were run to verify the system was not interacting on the timescale of the experiment. The weight average sedimentation coefficient showed no obvious trend with concentration (Figures S1 and S2) of fl-LOXL2 indicating a non-interacting system. A disperse distribution of four populations at monomer $(\sim 4.7 \mathrm{~s})$, dimer $(\sim 6.9 \mathrm{~s})$, tetramer $(\sim 9.3 \mathrm{~s})$, and pentamer $(\sim 11.5 \mathrm{~s})$ were observed (Figure $5 \mathrm{~A})$. Distribution integrations calculated were $83.6 \pm 1.1 \%$ of monomer, $11.6 \pm 0.3 \%$ of dimer and $4.7 \pm 1.5 \%$ of tetramer and pentamer together (Figure $5 \mathrm{~B}$ ). 
(A)

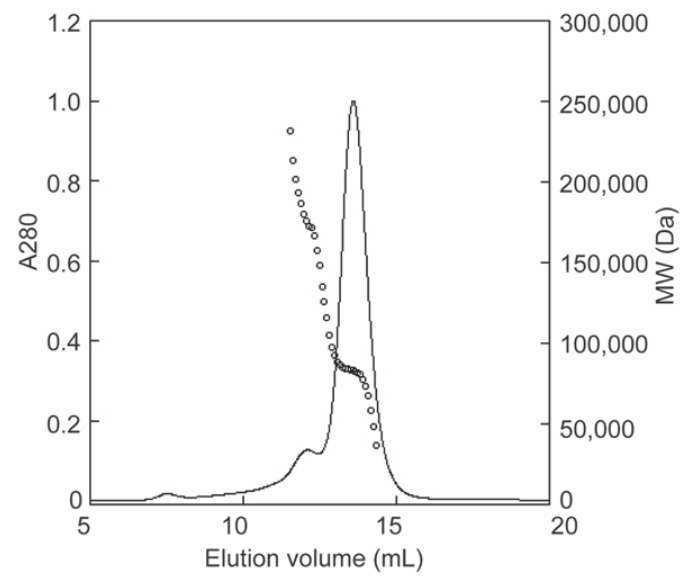

(B)

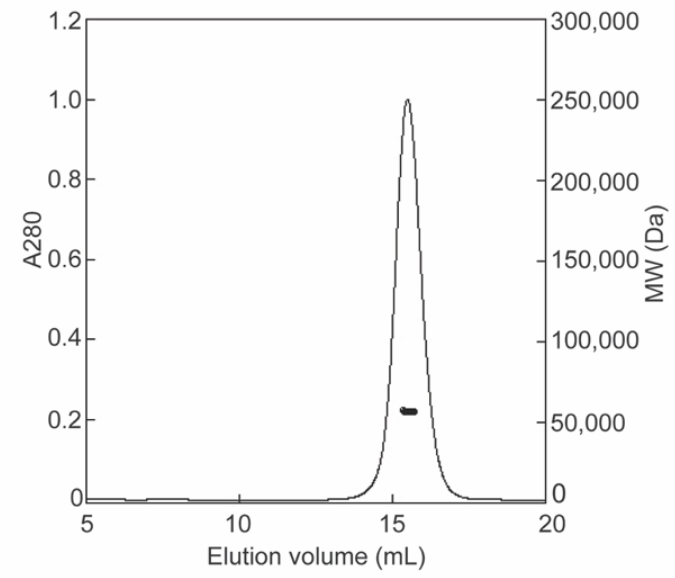

Figure 4. Molecular weight (MW) determination of LOXL2 by SEC-MALS (A) fl-LOXL2 (B) $\Delta 1$ 2SRCR-LOXL2; for clarity only every 10th measurement of MW is plotted.

(A)

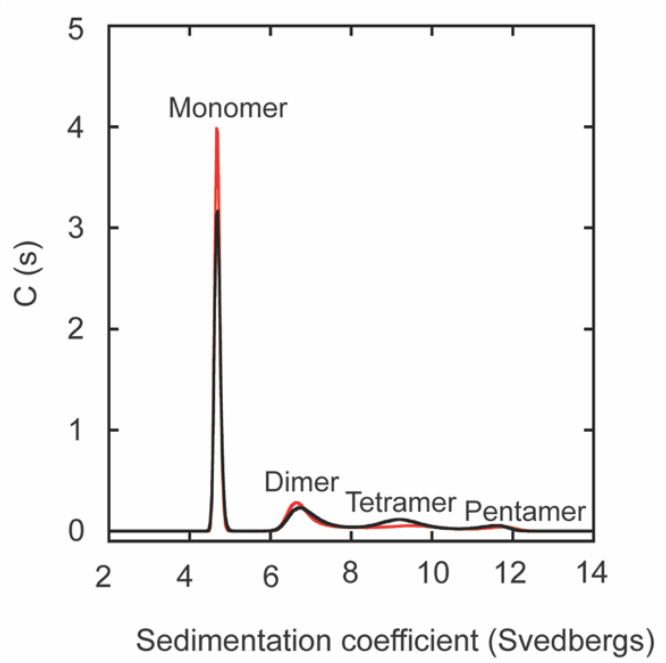

(B)

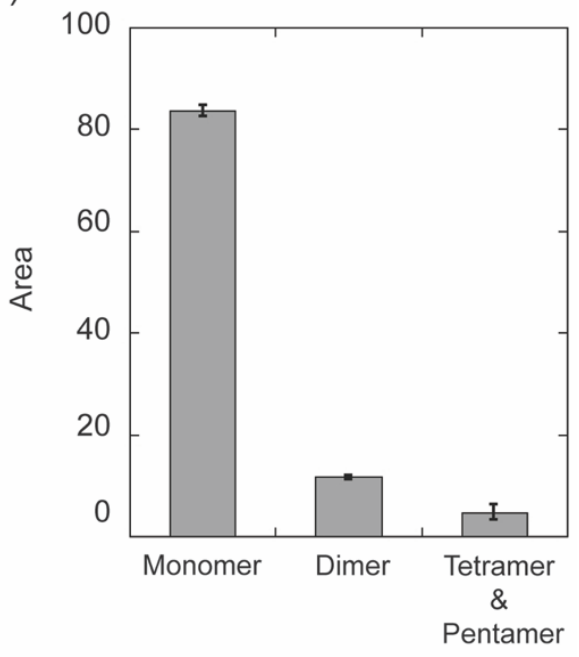

Oligomeric state

Figure 5. The oligomeric state of fl-LOXL2. (A) A representative run of SV-AUC with $0.88 \mathrm{mg} / \mathrm{mL}$ of fl-LOXL2. (B) A digitized graph showing the percentage of monomer, dimer, tetramer and pentamer of fl-LOXL2.

The shape of a molecule can be assessed from SV-AUC data by examining the frictional ratio, $\mathrm{f} / \mathrm{f}_{0}$, which can be thought of as a measure of asymmetry. $\mathrm{F} / \mathrm{F}_{0}$ is equal to $\mathrm{s}_{\max } / \mathrm{s}^{*}$, the ratio of the maximum theoretical sedimentation coefficient (that of a perfect sphere) to the observed sedimentation coefficient. If we use $\sim 86.2-\mathrm{kDa}$ for the monomer of LOXL2, the maximum of sedimentation coefficient can be calculated as $\sim 7.0 \mathrm{~s}$ (s: Svedberg unit, $10^{-13} \mathrm{~s}$ ) by using equation: $\mathrm{s}_{\max }=0.00361 \mathrm{M}^{\frac{2}{3}}$ ( $\mathrm{s}_{\max }$ : the maximum theoretical sedimentation coefficient, $\mathrm{M}=$ molecular weight) $[29,30]$. The observed sedimentation coefficient is $\sim 4.7 \mathrm{~s}$, that will make $\mathrm{f} / \mathrm{f}_{0}=\mathrm{s}_{\max } / \mathrm{s}^{*}=7.0 \mathrm{~s} / 4.7 \mathrm{~s}=1.5$. This indicates that fl-LOXL2 is a moderately asymmetrical protein (Table 1). From these data we can also calculate a Stokes radius (radius of the equivalent sphere that sediments at the same rate) of $4.5 \mathrm{~nm}$. 
Table 1. A comparison of biophysical data of fl-LOXL2 and $\triangle 1-2 S R C R-L O X L 2$.

\begin{tabular}{|c|c|c|c|c|c|c|c|}
\hline LOXL2 & $\mathrm{pH}$ & Methods & $\begin{array}{c}\text { MW } \\
\text { (kDa) }\end{array}$ & $\underset{(\mathrm{nm})}{R_{\mathrm{g}}}$ & $\underset{(\mathrm{nm})}{R_{\mathrm{h}}}$ & $\begin{array}{c}10^{13} \mathrm{~s} \\
(\mathrm{sec})\end{array}$ & $\mathbf{f} / \mathbf{f}_{0}$ \\
\hline fl- (a) & 8.0 & AUC/SEC-MALS & $83 / 91^{(f)}$ & - & $4.58 \pm 0.05$ & 4.7 & 1.49 \\
\hline$\Delta 1-2 S R C R-{ }^{(a)}$ & 8.0 & AUC/SEC-MALS & 57.3 & - & $3.40 \pm 0.15$ & - & - \\
\hline fl- (b) & - & Computation $(\mathrm{e})$ & 86.7 & 3.31 & 4.48 & 4.73 & 1.54 \\
\hline$\Delta 1-2 S R C R-(\mathrm{c})$ & 7.6 & Computation (e) & 49.6 & 2.41 & 3.12 & 3.94 & 1.29 \\
\hline fl- (d) & 7.4 & AUC/SEC-MALS & 92.6 & - & 5.6 & 5.15 & 1.44 \\
\hline fl- (d) & 4.7 & TEM & 93 & 4.7 & 6.6 & 5.52 & - \\
\hline
\end{tabular}

(a) Purified recombinant proteins used in this study. (b) 3D structure predicted by AlphaFold V2 [20]. (c) The monomer part of the X-ray crystal structure (PDB:5ZE3) without water molecules. (d) Purified recombinant protein [4]. (e) Computational calculation by HullRad [31].

(f) Including N-glycans.

\subsection{Hydrodynamic Radii $\left(R_{h}\right)$ of fl- and $\triangle 1-2 S R C R-L O X L 2$}

Hydrodynamic radii $\left(R_{\mathrm{h}}\right)$ of fl-LOXL2 and $\triangle 1-2$ SRCR-LOXL2 were calculated from the "on-line" dynamic light scattering measurement (Figure 6, Table 1) and the dynamic light scattering signal was analyzed by the method of cumulants [24]. The $R_{\mathrm{h}}$ value of fl-LOXL2 $(4.58 \pm 0.05 \mathrm{~nm})$ is comparable to that $(4.98 \pm 0.08 \mathrm{~nm})$ of aldolase, a globular protein that has been routinely used as the standard [31,32]. However, the molecular weight of aldolase is $158-\mathrm{kDa}$ that is larger than the monomer of fl-LOXL2 $(\sim 86-\mathrm{kDa})$. On the other hand, the $R_{\mathrm{h}}$ value of $\Delta 1-2 \mathrm{SRCR}-\mathrm{LOXL2}(3.40 \pm 0.15 \mathrm{~nm})$ is in good agreement with those (3.40-3.69 nm) of bovine serum albumin and human serum albumin which both have similar mass $(66.5-\mathrm{kDa})[31]$ to that of $\Delta 1-2 \mathrm{SRCR}-\mathrm{LOXL} 2(\sim 66 \mathrm{kDa})$. These results suggest that fl-LOXL2 is a moderately elongated protein but $\triangle 1-2 S R C R-L O X L 2$ is an approximately globular protein. While the difference $(\sim 1.2 \mathrm{~nm})$ between $R_{\mathrm{h}}$ values of fl-LOXL2 and $\triangle 1-2 S R C R-L O X L 2$ is significant, it is still considerably smaller than the difference in molecular weights $(\sim 20-\mathrm{kDa})$. Therefore, the difference cannot account for a fully extended conformation of the first two SRCR domains of fl-LOXL2 (SRCR1 and SRCR2).

(A)

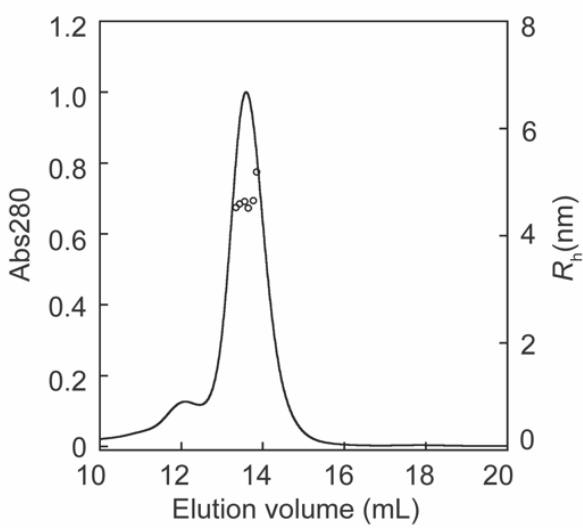

(B)

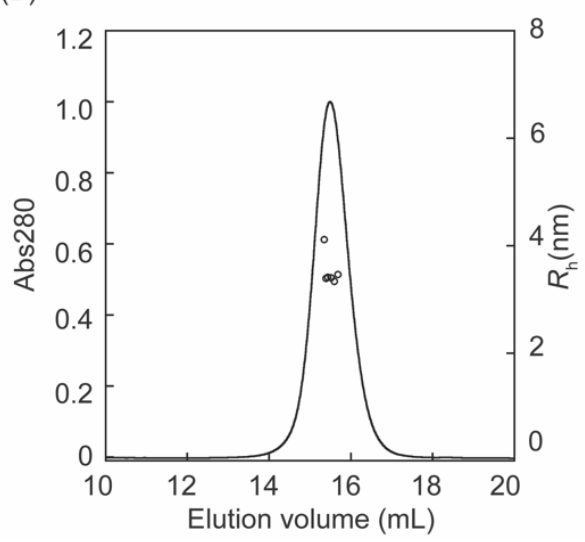

Figure 6. Hydrodynamic radii $\left(R_{\mathrm{h}}\right)$ of fl-LOXL2: (A) $\Delta 1$-2SRCR-LOXL2 (B) calculated from SECMALS analysis.

\subsection{Structure Prediction of $f l-L O X L 2$}

The overlaid image (Figure 7A) of the 3D structure of fl-LOXL2 (precursor form) that was predicted by AlphaFold 2 (AF-Q9Y4K0-F1-model_vi.pdb) [20] and X-ray crystal structure of $\triangle 1-2 S R C R-L O X L 2$ (precursor form) were generated by PyMOL [33]. The third and fourth SRCR domains and the C-terminal amine oxidase domain of the 3D modeled structure were superimposable with those of the X-ray structure, where the root mean square deviation (RMSD) for the overlay was calculated as $0.490 \AA$. The first and second SRCR domains (in slate and in purple, respectively) of the 3D modeled structure have domain-domain interactions with the third SRCR domain (in cyan) but not with the fourth 
SRCR domain (in green) (Figure 7B, left). As seen in the crystal structure of $\triangle 1-2 \mathrm{SRCR}$ LOXL2, there is no direct interaction between the third and fourth SRCR domains of the 3D predicted structure. The PACE4 cleavage site $\left({ }^{314} \mathrm{Arg}-{ }^{315} \mathrm{Phe}-{ }^{316} \mathrm{Arg}-{ }^{317} \mathrm{Lys}-\downarrow^{318} \mathrm{Ala}\right)[4]$ is in the loop region between the second and the third SRCR domains and solvent-exposed (Figure 7B, right). The three N-glycosylation sites (Asn288, Asn455, Asn644) [34] are all solvent-exposed as well. The $R_{\mathrm{h}}$ value of the 3D predicted structure of fl-LOXL2 was calculated as $4.48 \mathrm{~nm}$ by HullRad [31] that is within a reasonable margin of error of the $R_{\mathrm{h}}$ value $(4.58 \pm 0.05 \mathrm{~nm})$ calculated from our mature fl-LOXL2 in solution by SEC-MALS (Table 1). These results indicate that the overall domain organizations of the 3D modeled precursor fl-LOXL2 are closely related to the mature fl-LOXL2 in solution.

(A)

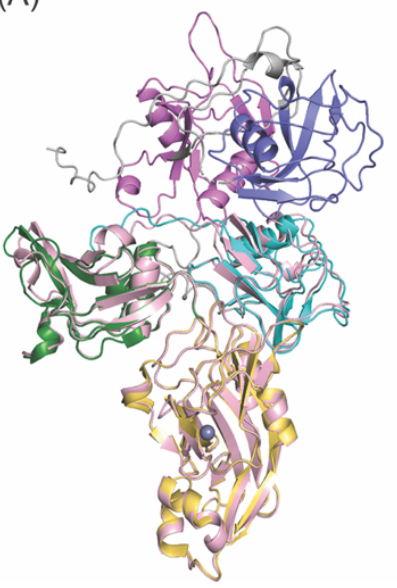

(B)

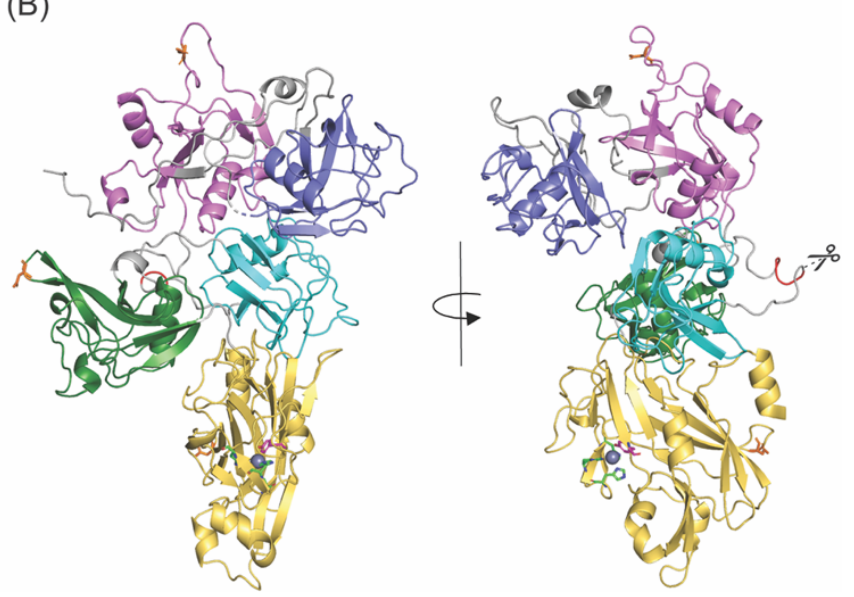

Figure 7. Structure prediction of fl-LOXL2. (A) Overlay of 3D structure of fl-LOXL2 predicted by AlphaFold ver 2 (SRCR1 in slate, SRCR2 in purple, SRCR3, SRCR4, amine oxidase domain in lightpink) and X-ray crystal structure of $\triangle 1$-2SRCR-LOXL2 (SRCR3 in cyan, SRCR4 in green, amine oxidase domain in yellow). $\mathrm{Zn}^{2+}$ occupying the $\mathrm{Cu}^{2+}$-binding site is shown as a gray sphere. (B) 3D structure of fl-LOXL2 predicted by AlphaFold ver 2 (SRCR 1 in slate, SRCR in purple, SRCR3 in cyan, SRCR4 in green, amine oxidase domain in yellow) in two angles. The PACE4 cleavage site $\left({ }^{314} \mathrm{Arg}-\right.$ ${ }^{315} \mathrm{Phe}^{316}{ }^{316} \mathrm{Arg}_{-}{ }^{317} \mathrm{Lys}_{-} \downarrow^{318} \mathrm{Ala}$ ) is highlighted in red. The $\mathrm{N}$-glycosylation sites (Asn288, Asn455, Asn644) are in orange stick. Peptides connecting domains are in gray.

During the course of our study, $R_{\mathrm{h}}$ values of $5.6-6.6 \mathrm{~nm}$ for a fl-LOXL2 were calculated from SEC-MALS and single-particle electron microscopy (EM), respectively [5]. In that study, a recombinant fl-LOXL2 was purified from the conditioned media of Chinese hamster ovary cells stably transfected with an expression construct of C-terminally His-tagged flLOXL2. The EM images after negative staining indicated that the four SRCR domains of the fl-LOXL2 are fully extended and there was no sign of domain-domain interactions. The $R_{\mathrm{h}}$ values determined for the fl-LOXL2 are 1-2 nm (10-20 Å) larger than those calculated for both fl-LOXL2 and the 3D predicted structure (AlphaFold V2) that have been previously discussed in this study (Table 1). As mentioned earlier, in our hands, the difference observed in $R_{\mathrm{h}}$ values $(1.18 \pm 0.02 \mathrm{~nm}$, or $11.8 \pm 0.2 \AA)$ between fl-LOXL2 and $\Delta 1-2 S R C R-L O X L 2$ (mature forms) are in good agreement with the difference in $R_{\mathrm{h}}$ values calculated $(1.36 \mathrm{~nm}$, 13.6 $\AA$ ) between the 3D-predicted structure of fl-LOXL2 and the X-ray crystal structure of the monomer of $\triangle 1-2 S R C R-L O X L 2$ (precursor forms) by HullRad [31]. Our results strongly suggest that the overall structures of both fl-LOXL2 and $\triangle 1$-2SRCR-LOXL2 (mature forms) in solution are related to those of 3D-predicted fl-LOXL2 and X-ray crystal structure of $\triangle 1$-2SRCR-LOXL2 (precursor forms). The difference in $R_{\mathrm{h}}$ value determined by EM may be due to the acidic environment ( $\mathrm{pH}$ 4.7) for staining. 


\subsection{The Isoelectric Point ( $p I)$ of LOXL2}

The isoelectric points (pIs) of a series of recombinant LOXL2s were determined by isoelectric focusing polyacrylamide gel electrophoresis (IEF-PAGE) (Table 2). The experimentally determined pIs are in good agreement with values predicted by Isoelectric Compute pI/MW [35] and Point Calculator 2.0 [36]. A slight smearing of fl-LOXL2 was detected and two major spots gave a pI of 5.5-5.9. This is most likely due to the heterogeneity of N-glycoconjugates (31 varieties at Asn288) [34]. Although N-glycoconjugates at Asn644 also possess 34 varieties [34], they did not have significant influence on pI values for all forms of recombinant LOXL2 in this study. Overall, LOXL2 is a weakly acidic protein. A surface electrostatic potential map generated by PyMOL with APBS Electrostatistics plug-in [37] shows a large acidic patch expanding from the amine oxidase domain through the SRCR3 domain to the SRCR1 domain in one face of fl-LOXL2 (Figure S3A). On the other hand, there is a basic patch present in the SRCR2 domain and another acidic patch in the amine oxidase domain on the other face of fl-LOXL2 (Figure S3B). The estimated pI value of the SRCR2 domain is 8.8-9.6 (Table 3), which is over $3 \mathrm{pH}$ unit more basic than other three SRCR domains. Further study is necessary to define the biological and physiological significance of the basicity of the SRCR2 domain.

Table 2. Isoelectric points (pIs) of the series of recombinant LOXL2s in this study.

\begin{tabular}{cc}
\hline LOXL2 & pI $\left(\right.$ Computed $\left._{\mathbf{p I}} \mathbf{(}^{(\mathbf{a}, \mathbf{b})}\right)$ \\
\hline fl-LOXL2 & $5.5-5.9(5.7,6.0)$ \\
$\Delta$ 1-2SRCR-LOXL2 & $5.4(5.9,5.9)$ \\
$\Delta$ 1-3SRCR-LOXL2 & $5.9(5.3,5.1)$ \\
$\Delta$ 1-4SRCR-LOXL2 & $5.1(5.1,4.9)$ \\
\hline
\end{tabular}

(a) Compute $\mathrm{pI} / \mathrm{Mw}$ [36], (b) Isoelectric Point Calculator 2.0 [37].

Table 3. Isoelectric points (pIs) of SRCR domains of fl-LOXL2.

\begin{tabular}{cc}
\hline SRCR Domain of LOXL2 & pI $^{\left(\text {Computed }^{(\mathbf{a}, \mathbf{b})}\right)}$ \\
\hline SRCR1 & $5.4,5.2$ \\
SRCR2 & $9.6,8.8$ \\
SRCR3 & $5.1,5.0$ \\
SRCR4 & $5.9,5.6$ \\
\hline
\end{tabular}

(a) Compute pI/Mw [36], (b) Isoelectric Point Calculator 2.0 [37].

A significant amount of dimerization of LOXL2 was only observed with fl-LOXL2 at concentrations $\geq 0.88 \mathrm{mg} / \mathrm{mL}$ (Figures 3 and 5 ). As the dimerization only occurred within fl-LOXL2, it is most likely that the dimerization involves one or both of the first two SRCR domains. Two docking programs, PatchDock and ZDOCK were used to generate possible modes of dimerization [38-40]. While the most common pattern seen was interaction between SRCR1 of monomer and SRCR2 of the other monomer (an example is shown in Figure S4), some models that were generated showed interactions between the first SRCR domain and the catalytic domain. More study is necessary, such as H/D exchange mass spectrometry, site-directed mutagenesis, or deletion of the binding motif to elucidate the nature of the dimerization of fl-LOXL2.

\section{Conclusions}

In solution, fl-LOXL2 exists predominantly as a monomer, but it can be in equilibrium with dimer-tetramer-pentamer when the protein concentration is $\geq 0.88 \mathrm{mg} / \mathrm{mL}$ (monomer: $83.6 \%$; dimer: $11.6 \%$; tetramer + pentamer: $4.7 \%$ ). On the other hand, $\Delta 1-$ 2SRCR-, $\triangle 1-3 S R C R-$, and $\triangle 1-4$ SRCR-LOXL2s exists exclusively as a monomer. These results suggest that the dimerization of fl-LOXL2 involves one or both of the first two SRCR domains. The pI value of the SRCR2 domain is basic ( 9.2), in contrast to the rest of the SRCR domains and the catalytic domain of LOXL2 (5 6). This basicity of the SRCR2 domain potentially mediates protein-protein interaction in the ECM or cell-surface 
through ionic interactions. The higher affinity of fl-LOXL2 to the ECM that we previously observed [4] could partly be attributed to the basicity of the SRCR2 domain. LOXL2 has been shown to oxidatively deaminate Lys residue(s) of the extracellular domain of PDGFR $\beta$ to an aldehyde [6]. The pI value of the extracellular domain of PDGFR $\beta$ is predicted to be 4.83 and 4.71 by Isoelectric Compute pI/MW [35] and Point Calculator 2.0 [36], respectively. It is possible that the interaction of LOXL2 and PDGFR $\beta$ is facilitated by the SRCR2 domain. The $R_{\mathrm{h}}$ values determined for fl-LOXL2 and $\Delta 1$-2SRCR-LOXL2 in solution strongly suggest that the overall structures of both fl-LOXL2 and $\triangle 1-2 S R C R-L O X L 2$ in solution are closely related to those of 3D-predicted fl-LOXL2 and X-ray crystal structure of $\Delta 1$-2SRCR-LOXL2, and the LTQ cofactor biogenesis does not have a significant effect on the overall structure of LOXL2.

Supplementary Materials: The following are available online at https: / / www.mdpi.com/article/ 10.3390/biom11121846/s1, Figure S1: Weight average sedimentation coefficient with concentration of fl-LOXL2, Figure S2: Data, fit, and residuals plot for a representative SV-AUC run of fl-LOXL2 at $0.88 \mathrm{mg} / \mathrm{mL}$, Figure S3: Electrostatic potential on the surface of 3D-predicted LOXL2, Figure S4: A predicted dimer of fl-LOXL2 generated by ZDOCK.

Author Contributions: Conceptualization, M.M.; methodology, data acquisition \& analyses, M.M., A.A.M., H.-J.M., R.T.IV, E.F.-S., K.K.; original draft preparation, M.M., A.A.M., R.T.IV, E.F.-S.; review \& editing, M.M., A.A.M., H.-J.M., R.T.IV, E.F.-S., K.K., C.R.M. All authors have read and agreed to the published version of the manuscript.

Funding: The National Institutes of Health (NIH) Grants R01GM113101, the Kansas Masonic Cancer Research Institute Pilot Research Program of the University of Kansas Cancer Center, P30CA168524, and the COBRE-PSF P30 GM110761 Pilot Project, the University of Kansas, Department of Chemistry (to M.M) provided funding for this research. A.M. was supported by National Institutes of Health NIGMS Biotechnology Predoctoral Training Program (T32-GM008359) and the J.K. Lee Summer Scholar Program from the Department of Chemistry, the University of Kansas. The SEC-MALS instrumentation was supported by NIH Grant 1S10RR023748 01 (to E.F.-S).

Institutional Review Board Statement: Not applicable.

Informed Consent Statement: Not applicable.

Data Availability Statement: The data within this article are available from the authors upon request but may require data transfer agreement.

Conflicts of Interest: The authors declare no conflict of interest.

\section{References}

1. Csiszar, K. Lysyl oxidases: A novel multifunctional amine oxidase family. Prog. Nucleic Acid Res. Mol. Biol. 2001, 70, 1-32.

2. Moon, H.J.; Finney, J.; Ronnebaum, T.; Mure, M. Human lysyl oxidase-like 2. Bioorg. Chem. 2014, 57, 231-241. [CrossRef]

3. Anazco, C.; Lopez-Jimenez, A.J.; Rafi, M.; Vega-Montoto, L.; Zhang, M.Z.; Hudson, B.G.; Vanacore, R.M. Lysyl Oxidase-like-2 Cross-links Collagen IV of Glomerular Basement Membrane. J. Biol. Chem. 2016, 291, 25999-26012. [CrossRef]

4. Okada, K.; Moon, H.J.; Finney, J.; Meier, A.; Mure, M. Extracellular processing of lysyl oxidase-like 2 and its effect on amine oxidase activity. Biochemistry 2018, 57, 6973-6983. [CrossRef]

5. Schmelzer, C.E.H.; Heinz, A.; Troilo, H.; Lockhart-Cairns, M.P.; Jowitt, T.A.; Marchand, M.F.; Bidault, L.; Bignon, M.; Hedtke, T.; Barret, A.; et al. Lysyl oxidase-like 2 (LOXL2)-mediated cross-linking of tropoelastin. FASEB J. 2019, 33, 5468-5481, fj201801860RR. [CrossRef]

6. Mahjour, F.; Dambal, V.; Shrestha, N.; Singh, V.; Noonan, V.; Kantarci, A.; Trackman, P.C. Mechanism for oral tumor cell lysyl oxidase like-2 in cancer development: Synergy with PDGF-AB. Oncogenesis 2019, 8, 34. [CrossRef]

7. Wang, C.; Xu, S.; Tian, Y.; Ju, A.; Hou, Q.; Liu, J.; Fu, Y.; Luo, Y. Lysyl Oxidase-Like Protein 2 Promotes Tumor Lymphangiogenesis and Lymph Node Metastasis in Breast Cancer. Neoplasia 2019, 21, 413-427. [CrossRef] [PubMed]

8. Dongiovanni, P.; Meroni, M.; Baselli, G.A.; Bassani, G.A.; Rametta, R.; Pietrelli, A.; Maggioni, M.; Facciotti, F.; Trunzo, V.; Badiali, S.; et al. Insulin resistance promotes Lysyl Oxidase Like 2 induction and fibrosis accumulation in non-alcoholic fatty liver disease. Clin. Sci. 2017, 131, 1301-1315. [CrossRef]

9. Kasashima, H.; Yashiro, M.; Kinoshita, H.; Fukuoka, T.; Morisaki, T.; Masuda, G.; Sakurai, K.; Kubo, N.; Ohira, M.; Hirakawa, K. Lysyl oxidase-like 2 (LOXL2) from stromal fibroblasts stimulates the progression of gastric cancer. Cancer Lett. 2014, 354, 438-446. [CrossRef] 
10. Klepfish, M.; Gross, T.; Vugman, M.; Afratis, N.A.; Havusha-Laufer, S.; Brazowski, E.; Solomonov, I.; Varol, C.; Sagi, I. LOXL2 Inhibition Paves the Way for Macrophage-Mediated Collagen Degradation in Liver Fibrosis. Front. Immunol. 2020, 11, 480. [CrossRef]

11. Leung, L.; Niculescu-Duvaz, D.; Smithen, D.; Lopes, F.; Callens, C.; McLeary, R.; Saturno, G.; Davies, L.; Aljarah, M.; Brown, M.; et al. Anti-metastatic Inhibitors of Lysyl Oxidase (LOX): Design and Structure-Activity Relationships. J. Med. Chem. 2019, 62, 5863-5884. [CrossRef] [PubMed]

12. Hutchinson, J.H.; Rowbottom, M.W.; Lonergan, D.; Darlington, J.; Prodanovich, P.; King, C.D.; Evans, J.F.; Bain, G. Small Molecule Lysyl Oxidase-like 2 (LOXL2) Inhibitors: The Identification of an Inhibitor Selective for LOXL2 over LOX. ACS Med. Chem. Lett. 2017, 8, 423-427. [CrossRef]

13. Rowbottom, M.W.; Bain, G.; Calderon, I.; Lasof, T.; Lonergan, D.; Lai, A.; Huang, F.; Darlington, J.; Prodanovich, P.; Santini, A.M.; et al. Identification of 4-(Aminomethyl)-6-(trifluoromethyl)-2-(phenoxy)pyridine Derivatives as Potent, Selective, and Orally Efficacious Inhibitors of the Copper-Dependent Amine Oxidase, Lysyl Oxidase-Like 2 (LOXL2). J. Med. Chem. 2017, 60, 4403-4423. [CrossRef]

14. Findlay, A.D.; Foot, J.S.; Buson, A.; Deodhar, M.; Jarnicki, A.G.; Hansbro, P.M.; Liu, G.; Schilter, H.; Turner, C.I.; Zhou, W.; et al. Identification and Optimization of Mechanism-Based Fluoroallylamine Inhibitors of Lysyl Oxidase-like 2/3. J. Med. Chem. 2019, 62, 9874-9889. [CrossRef]

15. Schilter, H.; Findlay, A.D.; Perryman, L.; Yow, T.T.; Moses, J.; Zahoor, A.; Turner, C.I.; Deodhar, M.; Foot, J.S.; Zhou, W.; et al. The lysyl oxidase like 2/3 enzymatic inhibitor, PXS-5153A, reduces crosslinks and ameliorates fibrosis. J. Cell. Mol. Med. 2019, 23, 1759-1770. [CrossRef] [PubMed]

16. Wong, C.C.; Tse, A.P.; Huang, Y.P.; Zhu, Y.T.; Chiu, D.K.; Lai, R.K.; Au, S.L.; Kai, A.K.; Lee, J.M.; Wei, L.L.; et al. Lysyl oxidase-like 2 is critical to tumor microenvironment and metastatic niche formation in hepatocellular carcinoma. Hepatology 2014, 60, 1645-1658. [CrossRef]

17. Martinez, V.G.; Moestrup, S.K.; Holmskov, U.; Mollenhauer, J.; Lozano, F. The conserved scavenger receptor cysteine-rich superfamily in therapy and diagnosis. Pharmacol. Rev. 2011, 63, 967-1000. [CrossRef]

18. Canton, J.; Neculai, D.; Grinstein, S. Scavenger receptors in homeostasis and immunity. Nat. Rev. Immunol. 2013, 13, 621-634. [CrossRef]

19. Zhang, X.; Wang, Q.; Wu, J.; Wang, J.; Shi, Y.; Liu, M. Crystal structure of human lysyl oxidase-like 2 (hLOXL2) in a precursor state. Proc. Natl. Acad. Sci. USA 2018, 115, 3828-3833. [CrossRef]

20. Jumper, J.; Evans, R.; Pritzel, A.; Green, T.; Figurnov, M.; Ronneberger, O.; Tunyasuvunakool, K.; Bates, R.; Zidek, A.; Potapenko, A.; et al. Highly accurate protein structure prediction with AlphaFold. Nature 2021, 596, 583-589. [CrossRef]

21. Xu, L.; Go, E.P.; Finney, J.; Moon, H.; Lantz, M.; Rebecchi, K.; Desaire, H.; Mure, M. Post-translational Modifications of Recombinant Human Lysyl Oxidase-like 2 (rhLOXL2) Secreted from Drosophila S2 Cells. J. Biol. Chem. 2013, 288, 5357-5363. [CrossRef] [PubMed]

22. Culpepper, M.A.; Scott, E.E.; Limburg, J. Crystal structure of prolyl 4-hydroxylase from Bacillus anthracis. Biochemistry 2010, 49, 124-133. [CrossRef] [PubMed]

23. Folta-Stogniew, E.; Williams, K.R. Determination of molecular masses of proteins in solution: Implementation of an HPLC size exclusion chromatography and laser light scattering service in a core laboratory. J. Biomol. Tech. 1999, 10, 51-63. [PubMed]

24. Koppel, D.E. Analysis of Macromolecular Polydispersity in Intensity Correlation Spectroscopy: The Method of Cumulants. J. Chem. Phys. 1972, 57, 4814-4820. [CrossRef]

25. Schuck, P. Size-distribution analysis of macromolecules by sedimentation velocity ultracentrifugation and lamm equation modeling. Biophys. J. 2000, 78, 1606-1619. [CrossRef]

26. Cole, J.L. Analysis of heterogeneous interactions. Methods Enzymol. 2004, 384, 212-232.

27. Grabowski, S.J. Understanding Hydrogen Bonds: Theoretical and Experimental Views; The Royal Society of Chemistry: Cambridge, UK, 2021.

28. Marangon, M.; Van Sluyter, S.C.; Waters, E.J.; Menz, R.I. Structure of haze forming proteins in white wines: Vitis vinifera thaumatin-like proteins. PLoS ONE 2014, 9, e113757. [CrossRef] [PubMed]

29. Halsall, H.B.; Wermeling, J.R. Sedimentation Coefficient, Frictional Coefficient, and Molecular Weight. J. Chem. Educ. 1982, 59, 1076-1078.

30. Erickson, H.P. Principles of Protein-Protein Association; IOP Publishing Ltd.: Bristol, UK, 2019.

31. Fleming, P.J.; Fleming, K.G. HullRad: Fast Calculations of Folded and Disordered Protein and Nucleic Acid Hydrodynamic Properties. Biophys. J. 2018, 114, 856-869. [CrossRef]

32. Stetefeld, J.; McKenna, S.A.; Patel, T.R. Dynamic light scattering: A practical guide and applications in biomedical sciences. Biophys. Rev. 2016, 8, 409-427. [CrossRef]

33. Delano, W.L. The PyMOL Molecular Graphics System, Version 2.4.2; Schödinger, LLC.: New York, NY, USA, 2010.

34. Go, E.P.; Moon, H.J.; Mure, M.; Desaire, H. A recombinant human LOXL2 secreted from HEK cells displays complex, acidic glycans at all three N-linked glycosylation sites. J. Proteome Res. 2018, 17, 1826-1832. [CrossRef] [PubMed]

35. Gasteiger, E.; Hoogland, C.; Gattiker, A.; Duvaud, S.; Wilkins, M.R.; Appel, R.D.; Bairoch, A. Protein Identification and Analysis Tools on the ExPASy Server. In The Proteomics Protools Handbook; Walker, J.M., Ed.; Humana Press: Totowa, NJ, USA, 2005.

36. Kozlowski, L.P. IPC 2.0: Prediction of isoelectric point and pKa dissociation constants. Nucleic Acids Res. 2021, 49, W285-W292. [CrossRef] 
37. Baker, N.A.; Sept, D.; Joseph, S.; Holst, M.J.; McCammon, J.A. Electrostatics of nanosystems: Application to microtubules and the ribosome. Proc. Natl. Acad. Sci. USA 2001, 98, 10037-10041. [CrossRef] [PubMed]

38. Duhovny, D.; Nussinov, R.; Wolfson, H.J. Efficient Unbound Docking of Rigid Molecules. Lect. Notes Comput. Sci. 2002, $2452,185-200$.

39. Schneidman-Duhovny, D.; Inbar, Y.; Nussinov, R.; Wolfson, H.J. PatchDock and SymmDock: Servers for rigid and symmetric docking. Nucl. Acids Res. 2005, 33, 363-367. [CrossRef]

40. Pierce, B.G.; Hourai, Y.; Weng, Z. Accelerating protein docking in ZDOCK using an advanced 3D convolution library. PLoS ONE 2011, 6, e24657. [CrossRef] 